${ }^{1}$ Banks, B. E. C., Damjanovic, V., and Vernon, C. A., Nature, 240, 147 (1972).

${ }^{2}$ Exner, O., Coll. Czech. Chem. Comm., 37, 1425 (1972).

${ }^{3}$ Rosenberg, B., Kemeny, G., Switzer, R. C., and Hamilton, T. C., Nature, 232, 471 (1971).

\section{Thermodynamic Compensation Rule}

Banks, Damjanovic and Vernon' have proposed that the thermodynamic compensation rule which is claimed to apply to the denaturation of proteins ${ }^{2,3}$ is the result of an experimental artefact and thus of no physical significance. In particular, by taking logarithms of the unimolecular rate equation, they obtain

$$
\log C-\log k T / h=\Delta S \ddagger / 2.303 R-\Delta H \ddagger / 2.303 T
$$

where $C$ is the rate constant of the reaction. Provided that $\log C$ does not vary very much, for a small range of temperatures the left side of equation (1) is approximately constant so that $\Delta S \ddagger$ and $\Delta H^{\ddagger}$ are linearly related. But the real point of physical significance is not so much that $\Delta S \ddagger$ and $\Delta H^{\ddagger}$ are linearly related, but that $\log C$ should vary so very little with $\Delta H^{\ddagger}$. In the particular case they consider, this is the result of the experimental difficulty of measuring more than a small range of values of $C$ and $T$ so that only those materials which apparently obey the compensation law are susceptible to investigation.

The compensation law is also observed, however, in the electrical conductivity of organic semiconductors ${ }^{4}$ and amorphous semiconductors ${ }^{5}$. In those cases the measurable range of conductivity, $\sigma$, extends over at least twenty orders of magnitude so that any constancy of $\sigma$ at a particular temperature would be of real physical significance. In the cases cited this critical temperature is much higher than the experimental range of temperatures investigated, so that at the experimental temperature, the left side of equation (1) is no longer constant. In the case of amorphous semiconductors the critical temperature is greater than $1,000^{\circ} \mathrm{C}$. No doubt with a suitably wide and uncorrelated variation in $\Delta H \ddagger$ and $\sigma$ it would be possible to group together materials such that they apparently obey a compensation law. It is, however, physically significant that the groupings so obtained correspond to the groupings that would have been made on chemical or structural grounds.

Although a number of explanations of the compensation law for conductivity have been proposed ${ }^{6-9}$, there remains the possibility that this is also an experimental artefact ${ }^{10}$. But it is clear that the particular explanation proposed by Banks et al. cannot be appropriate in this case.

M. R. BOON

Imperial Chemical Industries Limited,

Corporate Laboratory,

PO Box 11,

The Heath,

Runcorn,

Cheshire

Received November 29, 1972.

1 Banks, B. E. C., Damjanovic, V., and Vernon, C. A., Nature, 240, $147(1972)$

2 Sukhazor, B. L., and Likhtenshtein, G. I., Biofizika, 10, 935 (1965).

${ }^{3}$ Rosenberg, B., Kemeny, G., Switzer, R. C., and Hamilton, T. C., Nature, 232, 471 (1971).

${ }^{4}$ Eley, D. D., J. Polymer Sci., C17, 73 (1967).

5 Stuke, J., J. Non Cryst. Sol., 4, 1 (1970).

6 Green, M. E., J. Chem. Phys., 51, 3279 (1969).

7 Kemeny, G., and Rosenberg, B., J. Chem. Phys., 53, 3549 (1970).

8 Boon, M. R., Disc. Faraday Soc., 51, 223 (1971).

9 Roberts, G. G., J. Phys., C, 4, 3167 (1971)

10 Johnston, G. R., and Lyons, L. E., Phys. Stat. Sol., 37, K43 (1970).

\section{Compensation Effect and Experimental Error}

Banks, Damjanovic and Vernon ${ }^{1}$ suggest that the observed compensation effect in the rates of some biological processes might be wholly artefactual. Their argument is one which would also apply to systems of purely chemical interest and raises the question of whether, in the inevitable presence of experimental errors, a true compensation effect is ever observable.

I use the term compensation effect to describe the systematic variation of the pre-exponential factor, $A$, with the activation energy, $E$, in the Arrhenius equation

$$
k=A \exp (-E / R T)
$$

often observed for a series of similar reactions. Here $k$ is the specific rate constant, $T$ the temperature and $R$ the gas constant. When a variation is observed, it is usually ${ }^{2}$ found to have the form

$$
\log A=\frac{E}{2.303 R T_{\mathrm{s}}}+\log k_{0}
$$

the parameter $T_{\mathrm{s}}$ has units of temperature and corresponds to the temperature at which all the reactions of the series proceed at the same rate, $k_{0}$.

Cremer ${ }^{3}$ discussed the possibility that compensation effects may arise out of experimental errors. For simplicity I consider separately the effect of uncertainties in $E$ and $k$ on $\log A$. Rearranging equation (1) and taking logarithms

$$
\log A=\log k+\frac{E}{2.303 R T}
$$

For an uncertainty $\delta E$ in $E$ there will be an associated uncertainty $\delta \log A$ in $\log A$ :

$$
\log A \pm \delta \log A=\log k+\frac{E \pm \delta E}{2.303 R T}
$$

Comparing this with equation (2) it is seen that the range of

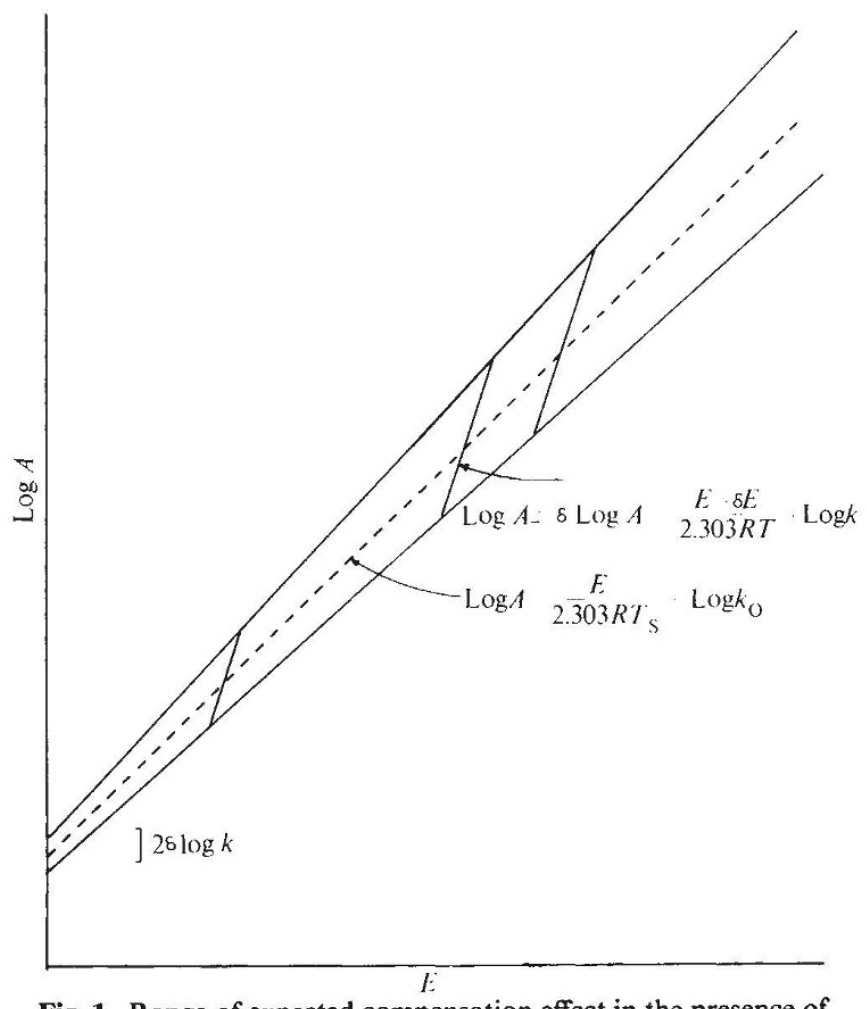

Fig. 1 Range of expected compensation effect in the presence of experimental error. 